\title{
QUOCIENTE DO DIÂMETRO PELA ÁREA BASAL E ZONAS DE COMPETIÇÃO EM POVOAMENTO MONOCLONAL DE Eucalyptus saligna
}

\author{
DIAMETER BY BASAL AREA RATIO AND COMPETITION ZONES IN \\ Eucalyptus saligna MONOCLONAL STAND \\ Paulo Renato Schneider ${ }^{1}$ César Augusto Guimarães Finger ${ }^{2}$ Paulo Sérgio Pigatto Schneider ${ }^{3}$ \\ Frederico Dimas Fleig ${ }^{4}$ Cláudio Thomas ${ }^{5}$ Jorge Antônio de Farias ${ }^{6}$
}

\begin{abstract}
RESUMO
O presente trabalho foi desenvolvido com o objetivo de estudar a relação do diâmetro e área basal, as zonas de competição, a densidade relativa e o autodesbaste em populações monoclonais de Eucalyptus saligna Smith, com base em 238 parcelas permanentes distribuídas em povoamentos com densidade completa, medidas dos 3 aos 18 anos de idade. Os resultados obtidos permitiram concluir que o modelo de densidade de Tang apresentou uma boa precisão e eficiência. O autodesbaste tem início quando a população atinge um diâmetro médio de $8 \mathrm{~cm}$, quando a densidade foi igual a $0,3446(34,46 \%)$, com linha do gradiente de 1,5818 (b). O máximo incremento corrente anual em diâmetro ocorreu entre as idades de 14 e 15 anos quando as densidades eram de 12,5 e $25 \%$ caracterizando a zona de crescimento livre de competição entre os indivíduos. A zona de aumento da competição entre as densidades relativas de 25 e $50 \%$ e a zona de iminente mortalidade ocorreu entre as densidades relativas de 50 e $100 \%$. O modelo permitiu estabelecer em relação à densidade relativa, cinco zonas de concorrência no desenvolvimento da população, definidas por zonas de espaço excessivo, crescimento livre, aumento de competição, estoque completo e iminente mortalidade.
\end{abstract}

Palavras-chave: densidade relativa; crescimento; autodesbaste.

\begin{abstract}
The present work was developed in order to study the relation among the diameter and the basal area, the competition zones, the relative density, and the self-tinning in Eucalyptus saligna Smith monoclonal stand, with 238 pair's data basis of permanent sampling by full density stand distribution, observed from 3 to 18 years old. The results obtained allowed to conclude that Tang's density model was very precise and efficient. The self-thinning begins when the population reaches an average diameter of $8 \mathrm{~cm}$. The relative density was equal $34.46 \%$, with line gradient of 1.5818 (b). The maximum current annual increment in
\end{abstract}

1. Engenheiro Florestal, Dr., Professor Titular do Departamento de Ciências Florestais, Centro de Ciências Rurais, Universidade Federal de Santa Maria, Av. Roraima, 1000, CEP 97105-900, Santa Maria (RS). 1977psps@gmail.com

2. Engenheiro Florestal, Dr., Professor Associado do Departamento de Ciências Florestais, Centro de Ciências Rurais, Universidade Federal de Santa Maria, Av. Roraima, 1000, CEP 97105-900, Santa Maria (RS). finger@smail.ufsm.br

3. Engenheiro Florestal, M.Sc., Doutorando do Programa de Pós-graduação em Engenharia Florestal do Centro de Ciências Rurais, Universidade Federal de Santa Maria, Av. Roraima, 1000, CEP 97105-900, Santa Maria (RS). psps@mail.ufsm.br

4. Engenheiro Florestal, Dr., Professor Adjunto do Departamento de Ciências Florestais, Centro de Ciências Rurais, Universidade Federal de Santa Maria, Av. Roraima, 1000, CEP 97105-900, Santa Maria (RS). ffleig@smail.ufsm.br

5. Engenheiro Florestal, M.Sc., Doutorando do Programa de Pós-graduação em Engenharia Florestal do Centro de Ciências Rurais, Universidade Federal de Santa Maria, Av. Roraima, 1000, CEP 97105-900, Santa Maria (RS). ctomas@mail.ufsm.br

6. Engenheiro Florestal, Dr., Professor Adjunto do Departamento de Ciências Florestais, Centro de Ciências Rurais, Universidade Federal de Santa Maria, Av. Roraima, 1000, CEP 97105-900, Santa Maria (RS). fariasufsm@gmail.com

Recebido para publicação em 6/04/2010 e aceito em 27/08/2010 
diameter and between the relative densities was observed between the ages of 14 and 15 years when the relative densities were $12.5 \%$ and $25 \%$, characterizing a free growth competition zone among the individuals of the population. The zone of increasing competition between the relative densities of 25 and $50 \%$ and the imminent mortality zone occurred between the relative densities of 50 and $100 \%$. The density model allowed detecting five competition zones, in the development of the stands, defined by zones of: excessive space, free growth, increase of competition, complete stock and imminent mortality, in relation to the relative density of the population.

Keywords: relative density; growth; self-thinning.

\section{INTRODUÇÃO}

O processo de competição intraespecífica estabelece em algum momento o autodesbaste no povoamento florestal, mas somente a sua determinação é insuficiente para otimização do manejo dos povoamentos. A competição intraespecífica é um importante fator no manejo por afetar diretamente o tamanho médio das plantas. Por esse motivo muitos estudos têm sido realizados visando descrever a relação entre o tamanho das plantas e a densidade da população, bem como a sua evolução no tempo.

As árvores de um plantio equiâneo e puro expandem suas copas tanto no sentido horizontal como no vertical para interceptar maior quantidade de energia solar, o que repercute na competição entre os indivíduos com o aumento da idade, culminando com a dominação e a consequente mortalidade de árvores. A supressão de indivíduos seguida pela mortalidade natural sem intervenção antrópica é convencionalmente chamada de autodesbaste.

$\mathrm{O}$ conceito de densidade de manejo resumese num processo de controle do crescimento do estoque inicial e do remanescente de desbastes visando os objetivos estabelecidos para a produção de matéria-prima. A determinação do estoque de crescimento ideal é um processo complexo com envolvimento biológico, tecnológico e de fatores econômicos específicos para uma situação particular de manejo.

De acordo com Reineke (1933), em povoamento com densidade completa, a relação entre o número de árvores por hectare $(\mathrm{N})$ e o diâmetro quadrático médio das árvores $\left(\mathrm{d}_{\mathrm{g}}\right)$ é linear numa escala logarítmica, expressa por: $\mathrm{N}=\mathrm{a}_{\mathrm{g}} \mathrm{d}^{b}$. $\mathrm{O}$ autor considera que os parâmetros ( $\mathrm{a}$ e b) da equação são independentes da idade e qualidade do sítio e postulou que a relação entre o número de árvores por hectare e o diâmetro médio quadrático em povoamento sem tratamento é uma linha reta com um valor do coeficiente angular constante de -1,605. No entanto, Del Rio et al. (2001) constataram uma variação do coeficiente angular com a espécie e muitas vezes com a idade, com valores variando entre $-2,33$ e -1,54. Por outro lado, DeBell e Whitesell (1988) utilizaram o modelo de Reineke, com coeficiente igual a $-1,6$, para determinar a densidade de árvores por diâmetro de Eucalyptus saligna no Havaí.

Curtis (1982) utilizou-se do princípio definido por Reineke (1933) para descrever a relação entre a área basal $(\mathrm{G})$ com o diâmetro quadrático médio $\left(\mathrm{d}_{\mathrm{g}}\right)$, através da expressão: $\mathrm{G}=\mathrm{a} \cdot\left(\mathrm{d}_{\mathrm{g}}^{\mathrm{b}}\right)$. $\mathrm{O}$ autor encontrou para povoamentos de Pseudotsuga menziensii o valor do gradiente (b) entre os limites de 0,45 e 0,5 , sugerindo o uso de 0,5 para determinar a densidade relativa absoluta do povoamento. Para obter a linha de autodesbaste em regenerações naturais equiâneas de Eucalyptus delegatensis e Eucalyptus regnans, Borough et al. (1984) sugeriram o gradiente igual a $-0,667$ e $-0,70$, respectivamente. Estes valores são próximos ao proposto por Reineke $(-0,625)$ e muito semelhantes ao encontrado por Curtis (1982) para Pseudotsuga menziensii $(-0,667)$. Para qualquer dos gradientes, a linha de autodesbaste independe da qualidade do sítio e manejo sendo somente a posição desta linha descrita pela constante, mas varia com a espécie, pois indica sua relativa tolerância a competição.

Yoda (1963) adicionou na equação de densidade de Reineke um termo quadrático do diâmetro $\left(\mathrm{d}_{\mathrm{g}}\right)$ para representar a distribuição de densidade de árvores $(\mathrm{N})$ em função do diâmetro médio de área basal $\left(\mathrm{d}_{\mathrm{g}}\right)$, tendo resultado a expressão: $\ln (\mathrm{N})=\mathrm{b}_{0}-\mathrm{b}_{1} \cdot \ln \left(\mathrm{d}_{\mathrm{g}}\right)+\mathrm{b}_{2} \cdot\left(\ln \left(\mathrm{d}_{\mathrm{g}}\right)\right)^{2}$.

A lei do autodesbaste, da potência de valor $-3 / 2$, formulada por Yoda (1963), descreve a proporção máxima do número médio de árvores pelo tamanho de uma dada espécie, sendo a mortalidade dependente da densidade. Essa relação entre o logaritmo do volume médio das árvores e o número de árvores por hectare é linear, com coeficiente angular de $-1,5$ e descreve o número máximo de árvores de uma determinada dimensão, com idêntica taxa de crescimento em todos os locais, sendo que o autodesbaste deve ocorrer

Ci. F1., v. 21, n. 4, out.-dez., 2011 
quando as árvores tiverem uma completa ocupação do sítio. Esta lei inicialmente foi descrita por: $\ln (\mathrm{w})=\ln (\mathrm{C})-(3 / 2) \cdot \ln (\mathrm{N})$, em que, $\mathrm{N}$ é o número de árvores por hectare, w é o peso médio, $\mathrm{C}$ é a constante de proporcionalidade.

Tang et al. (1994), estenderam a teoria do autodesbaste para povoamentos em densidade completa e subestocados. Os autores constataram que o autodesbaste começa antes do povoamento atingir a máxima densidade e que a taxa de autodesbaste incrementa com a densidade atingindo o máximo na densidade máxima da população. No modelo, a lei do autodesbaste pode ser considerada um caso especial, pois permite obter para um diâmetro qualquer, a densidade e a produção sendo expressa por:

$$
\ln N(t)=\ln S_{f}-\frac{1}{\gamma} \ln \left[\left(d(t) / d_{o}\right)^{\beta \gamma}+\delta\right]
$$

Em que, té a idade do povoamento (ano); $\beta$ é a taxa máxima de autodesbaste sobre o povoamento de densidade completa; $\mathrm{N}(\mathrm{t})$ é a densidade do povoamento no tempo $\mathrm{t}$ (número de árvores por hectare); $\mathrm{d}(\mathrm{t})$ é o diâmetro médio das árvores no tempo $\mathrm{t}(\mathrm{cm}) ; \mathrm{S}_{\mathrm{f}}$ é índice mínimo de densidade do povoamento; $\gamma$ é o índice de autodesbaste; e, $d_{o}$ é o diâmetro básico padrão $(25 \mathrm{~cm}) . \mathrm{O} \delta$ é uma constante dependente da integração dos valores iniciais de densidade do povoamento $\left(\mathrm{N}_{1}\right)$ e do diâmetro médio inicial $\left(\mathrm{d}_{1}\right)$, sendo obtida pela relação:

$$
\delta=\left(S_{f} / N_{1}\right)^{\gamma}-\left(d_{1} / d_{o}\right)^{\beta \gamma}
$$

A lei do autodesbaste tem sido objeto de pesquisas e de discussões acerca da sua eficiência por vários pesquisadores, tais como: Reineke (1933); Yoda et al. (1963); Westoby (1981), West e Bourough (1983); Zeide (1985, 1987 e 1991); Smith e Hann (1986); Laasasenaho e Koivuniemi (1990), Sterba e Monserud (1993); Tang et al. (1994); Osawa e Allen (1993); Tang et al. (1995); Del Rio et al. (2001), entre outros.

Reid (2006) utilizou dados de plantações de Eucalyptus sp. e Pinus sp. da Austrália e Nova Zelândia para demonstrar a utilização do quociente determinado entre o diâmetro médio das árvores e a área basal do povoamento ( $\overline{\mathrm{d}}: \mathrm{G})$ no manejo de florestas não industriais de pequenas propriedades, tendo concluído que esse quociente é simples e prático para guiar a taxa de estoque de plantações de Eucalyptus. A manutenção desse quociente constante leva, consequentemente, a maior competição entre os indivíduos com o aumento da idade.

Por outro lado, Bredenkamp e Burkhart (1990), estudando o índice de espaçamento para Eucalyptus grandis, comprovaram a existência de um problema na definição do limite de densidade para a espécie, em decorrência da propriedade do gênero Eucalyptus em continuar crescendo mesmo que em severa competição da copa. Os autores encontraram um valor de 0,05 para o índice de densidade relativa usado para determinar a linha de autodesbaste da espécie, considerando que a mortalidade pela competição ocorreu somente em parcelas de alta densidade. Também, constataram que o coeficiente angular de valor igual a $-3 / 2$ da lei do autodesbaste pode desviar-se dentro dos limites de -3,75 até -0,96 em decorrência da dinâmica dos fatores que influem sobre o povoamento como a idade, espécie e qualidade do sítio.

O objetivo deste estudo, conduzido com dados de povoamento monoclonal de Eucalyptus saligna Smith, foi o de avaliar a precisão e eficiência dos modelos de densidade populacional em função do diâmetro, determinar a relação do crescimento diamétrico com o estabelecimento do autodesbaste e relacionar o quociente do diâmetro pela área basal com o autodesbaste.

\section{MATERIAL E MÉTODOS}

Os dados utilizados nesta pesquisa tiveram origem em áreas de reflorestamentos localizados a $30^{\circ} 20^{\prime}$ de latitude sul e 51⒊' de longitude oeste do meridiano de Greenwich, constituída de povoamentos do clone 4013 de Eucalyptus saligna Smith, distribuídos na região da Serra do Sudeste do estado do Rio Grande do Sul.

O clima geral na região é caracterizado como Cfa, mesotérmico subtropical, com verões quentes e sem estação seca com precipitação média anual de $1.500 \mathrm{~mm}$, com máximas de precipitação ocorrendo nos meses de julho, agosto e setembro. A temperatura média anual é de $16^{\circ} \mathrm{C}$, com temperatura média do mês mais quente de $20^{\circ} \mathrm{C}$ e do mês mais frio de $10^{\circ} \mathrm{C}$ (MORENO, 1961).

Os solos encontrados nesta região, conforme a classificação existente são cambissolos e neossolos, podendo ocorrer gleissolos e planossolos, característicos da região litorânea e, também, nitossolos e argissolos (EMBRAPA, 1999). 
Os dados dendrométricos utilizados têm origem em parcelas permanentes instaladas em povoamentos manejados emalto fuste, com desbaste, sob manejo convencional e, de experimento de desbaste instalado no Horto Florestal Barba Negra, de propriedade do grupo Celulose Riograndense. Nos dois casos a implantação da floresta ocorreu em 1991, em espaçamento 3,0m x 2,0m, perfazendo uma densidade média de 1.666 árvores por hectare.

Parte dos dados utilizados foi originada de um experimento de desbaste instalado com delineamento experimental em blocos ao acaso, com 5 tratamentos e 3 repetições, definidos por: tratamento 1: testemunha-sem desbaste; tratamento 2: desbaste para um índice de espaçamento relativo de $18 \%$; tratamento 3: desbaste para um índice de espaçamento relativo de $23 \%$; tratamento 4 : desbaste para um índice de espaçamento relativo de 28\%; tratamento 5: desbaste para um índice de espaçamento relativo de $33 \%$.

Os desbastes foram realizados quando o índice de espaçamento relativo atingiu o valor inferior a 16\%, executado por baixo, sendo eliminadas as árvores dominadas, doentes, mortas, com forma defeituosa e copa reduzida, bem como foi observada a distribuição espacial dos indivíduos remanescentes do povoamento. Após o primeiro desbaste foi realizada uma desrama de modo a manter a copa em cerca de $40 \%$ da altura total das árvores, executadas até uma altura de 8,5 metros de fuste livre de galhos.

As parcelas, de dimensões de $30 \mathrm{~m} \times 21 \mathrm{~m}$, perfazendo $630 \mathrm{~m}^{2}$ de área útil tinham bordadura de uma linha de árvores. A área total das parcelas era de $972 \mathrm{~m}^{2}$, com limites de $36 \mathrm{~m}$ x $27 \mathrm{~m}$. Cada parcela foi identificada por plaquetas de alumínio, sendo registrado o número do bloco, número da parcela e tratamento aplicado. Estas foram medidas periodicamente, sendo tomado o diâmetro, a altura, caracterizado o fuste e a posição sociológica das árvores no dossel.

De forma complementar, também foi utilizado dado dendrométrico de 238 parcelas permanentes de densidade completa, estratificadas em sítios naturais sobre povoamentos comerciais dessa espécie, implantados no espaçamento de $3 \mathrm{~m}$ $\mathrm{x} 2 \mathrm{~m}$.

A relação da densidade de árvores pelo diâmetro foi estudada através do número de árvores por hectare e o correspondente diâmetro médio das árvores do povoamento sendo testadas as equações: a) Modelo da densidade e diâmetro de Reineke (1933)

$$
\ln (N)=b_{0}-b_{1} \cdot \ln (d)
$$

b) Modelo da densidade e diâmetro de Yoda et al. (1963)

$$
\ln (N)=b_{0}-b_{1} \cdot \ln (d)-b_{2} \cdot(\ln (d))^{2}
$$

d) Modelo do autodesbaste de Tang et al.

$$
\ln N(t)=\ln S_{f}-\frac{1}{\gamma} \ln \left[\left(d(t) / d_{o}\right)^{\beta \gamma}+\delta\right]
$$

Em que, dé o diâmetro médio quadrático das árvores; h a altura média; $\mathrm{N}$ é o número de árvores por hectare; t é a idade do povoamento (ano); $\beta$ é a taxa máxima de autodesbaste sobre o povoamento de densidade completa; $\mathrm{N}(\mathrm{t})$ é a densidade do povoamento no tempo $\mathrm{t}$ (número de árvores por hectare); $\mathrm{d}(\mathrm{t})$ é o diâmetro médio das árvores no tempo $\mathrm{t}(\mathrm{cm}) ; \mathrm{S}_{\mathrm{f}}$ é o índice de máxima densidade do povoamento; $\gamma$ é o índice de autodesbaste; $d_{o}$ é o diâmetro básico padrão $(25 \mathrm{~cm})$. A constante $\delta$ é determinada pela densidade inicial do povoamento $\left(\mathrm{N}_{1}\right)$ e o diâmetro médio inicial $\left(\mathrm{d}_{1}\right)$, na seguinte fórmula:

$$
\delta=\left(S_{f} / N_{1}\right)^{\gamma}-\left(d_{1} / d_{o}\right)^{\beta \gamma}
$$

$\mathrm{O}$ quociente do diâmetro pela área basal do povoamento $(\overline{\mathrm{d}}: \mathrm{G})$ foi determinado pela relação entre a área basal $(\mathrm{G})$ e o diâmetro médio $(\overline{\mathrm{d}})$, sendo usando a expressão definida por Curtis (1982): $\mathrm{G}=\mathrm{a} \cdot\left(\overline{\mathrm{d}}^{\mathrm{b}}\right)$. E a medição da densidade relativa (DR) foi determinada pela expressão: $\mathrm{RD}=\mathrm{G} /\left(\overline{\mathrm{d}}^{\mathrm{b}}\right)$.

A validação dos modelos de densidade de árvores por hectare pelo diâmetro médio do povoamento foi realizada através dos critérios estatísticos, recomendados por Palahi et al. (2002) e Cellini et al. (2002) definidos pela tendência (bias), eficiência, coeficiente de determinação ajustado, erro padrão da estimativa e coeficiente de variação.

As regressões lineares foram ajustadas pelo procedimento PROC REG e as não lineares pelo procedimento PROC NLIN com o método GaussNewton, no pacote Statistical Analysis System SAS (1999).

\section{RESULTADOS E DISCUSSÕES}

Na Tabela 1 foram relacionados os dados 
dendrométricos médios empregados totalizando 187 pares de dados originados de parcelas permanentes em densidade completa, medidas com periodicidade variada entre as idades de 3 a 18 anos. O diâmetro médio da amostra foi igual a $17,8 \mathrm{~cm}$, com valor mínimo de $10,6 \mathrm{~cm}$ e máximo de $28,2 \mathrm{~cm}$. A área basal média foi de $33,9 \mathrm{~m}^{2} / \mathrm{ha}$, com variação de 11,2 a $74,1 \mathrm{~m}^{2} / \mathrm{ha}$, com densidade populacional média de 1.258 , variando de 1.019 a 1.643 indivíduos por hectare.

Os resultados estatísticos das equações de densidade de árvores por hectare em função do diâmetro médio encontram-se discriminados na Tabela 2.

Comparativamente, os resultados da Tabela 2 mostram que o modelo de Tang apresentou resultados estatísticos que espelham uma melhor precisão, com coeficiente de determinação de 0,95 , erro padrão da estimativa de 0,1026 e coeficiente de variação igual a 1,39. Por outro lado, a análise da eficiência (E) definiu o modelo de Tang com parâmetro estatístico igual a 0,2903 como o melhor, assim como em relação a Bias com valor de $-0,0534$.

Os valores residuais de densidade de árvores por hectare em função do diâmetro, em escala logarítmica, obtida no modelo de Tang, são apresentados na Figura 1. Observa-se uma regularidade da distribuição dos resíduos, indicando sua adequação para a estimativa do número de árvores por hectare em função do diâmetro médio da população. Isso aliado a precisão e praticidade do modelo confirmaram a seleção modelo de Tang.
Porém, deve-se ressaltar que o valor do coeficiente $\beta$ igual a 0,3322 difere grandemente do valor de $-3 / 2$ definido por Tang como indicativo do autodesbaste para espécies florestais. Isto indica que o autodesbaste nos povoamentos deste clone apresenta comportamento totalmente distinto do normalmente citado na literatura para outras espécies e de Eucalyptus sp., para outras regiões do mundo. Isto pode ser verificado pelo valor do parâmetro do gradiente que expressa a média de indivíduos nestas populações monoclonais de Eucalyptus saligna, representada pela linha média de densidade de árvore por hectare em função do diâmetro na Figura 2.

A partir da linha média, que representa a dinâmica média da densidade populacional com o crescimento diamétrico, foi estabelecida a linha que representa a máxima taxa de estoque de árvores por hectare na população e o momento em que ocorre o autodesbaste no povoamento. Estas linhas mostraram coerência com a linha de máximo obtida com parcelas permanentes com máxima densidade de árvores por hectare. Esta linha de máxima densidade constitui-se numa situação indesejada para o manejo, indicando que as intervenções de desbaste deveriam ser antecipadas para o momento da ocorrência da mortalidade natural. Na Figura 2, observa-se que o autodesbaste tem início, quando o povoamento atinge um diâmetro médio em torno de $8 \mathrm{~cm}$, indicando ser este momento em que começa a haver mortalidade de indivíduos, devido à concorrência por elementos essenciais comoluz,água

TABELA 1: Dados dendrométricos das parcelas permanentes de povoamentos de Eucalyptus saligna, clone 4013.

TABLE 1: Dendrometric data of the permanent sampling of Eucalyptus saligna, clone 4013.

\begin{tabular}{c|c|c|c|c|c}
\hline Variável & $\mathrm{N}^{\circ}$ Obs. & Média & Desvio padrão & Mínimo & Máximo \\
\hline $\mathrm{d}$ & 187 & 17,8 & 3,7 & 10,3 & 28,2 \\
\hline $\mathrm{G}$ & 187 & 33,9 & 11,27 & 11,2 & 74,1 \\
\hline $\mathrm{N}$ & 187 & 1258 & 154,29 & 1019 & 1643 \\
\hline
\end{tabular}

Em que: $\mathrm{d}=$ diâmetro médio $(\mathrm{cm}) . \mathrm{G}=$ área basal $\left(\mathrm{m}^{2} / \mathrm{ha}\right) ; \mathrm{N}=$ número de árvores (ha).

TABELA 2: Estatísticas dos modelos de densidade para Eucalyptus saligna, clone 4013.

TABLE 2: $\quad$ Statistical of tree density models for Eucalyptus saligna stands, clone 4013.

\begin{tabular}{|c|c|c|c|c|c|c|c|c|}
\hline \multirow{2}{*}{ Modelos } & \multicolumn{3}{|c|}{ Parâmetros } & \multirow{2}{*}{$\mathrm{R}^{2}$} & \multirow[b]{2}{*}{$S_{y x}$} & \multirow{2}{*}{$\mathrm{CV}(\%)$} & \multirow{2}{*}{ Bias } & \multirow{2}{*}{ E } \\
\hline & $\mathrm{S}_{f} / \mathrm{b}_{0}$ & $\beta / b_{1}$ & $\gamma / b_{2}$ & & & & & \\
\hline Tang & 1110,0 & 0,3322 & 1406,0 & 0,95 & 0,1026 & 1,39 & $-0,0534$ & 0,2903 \\
\hline Reineke & 8,0314 & $-0,3321$ & & 0,28 & 0,1028 & 1,44 & $-0,0934$ & 0,4490 \\
\hline Yoda & 8,5850 & $-0,6905$ & 0,0634 & 0,28 & 0,1030 & 1,44 & $-0,0865$ & 0,4495 \\
\hline
\end{tabular}




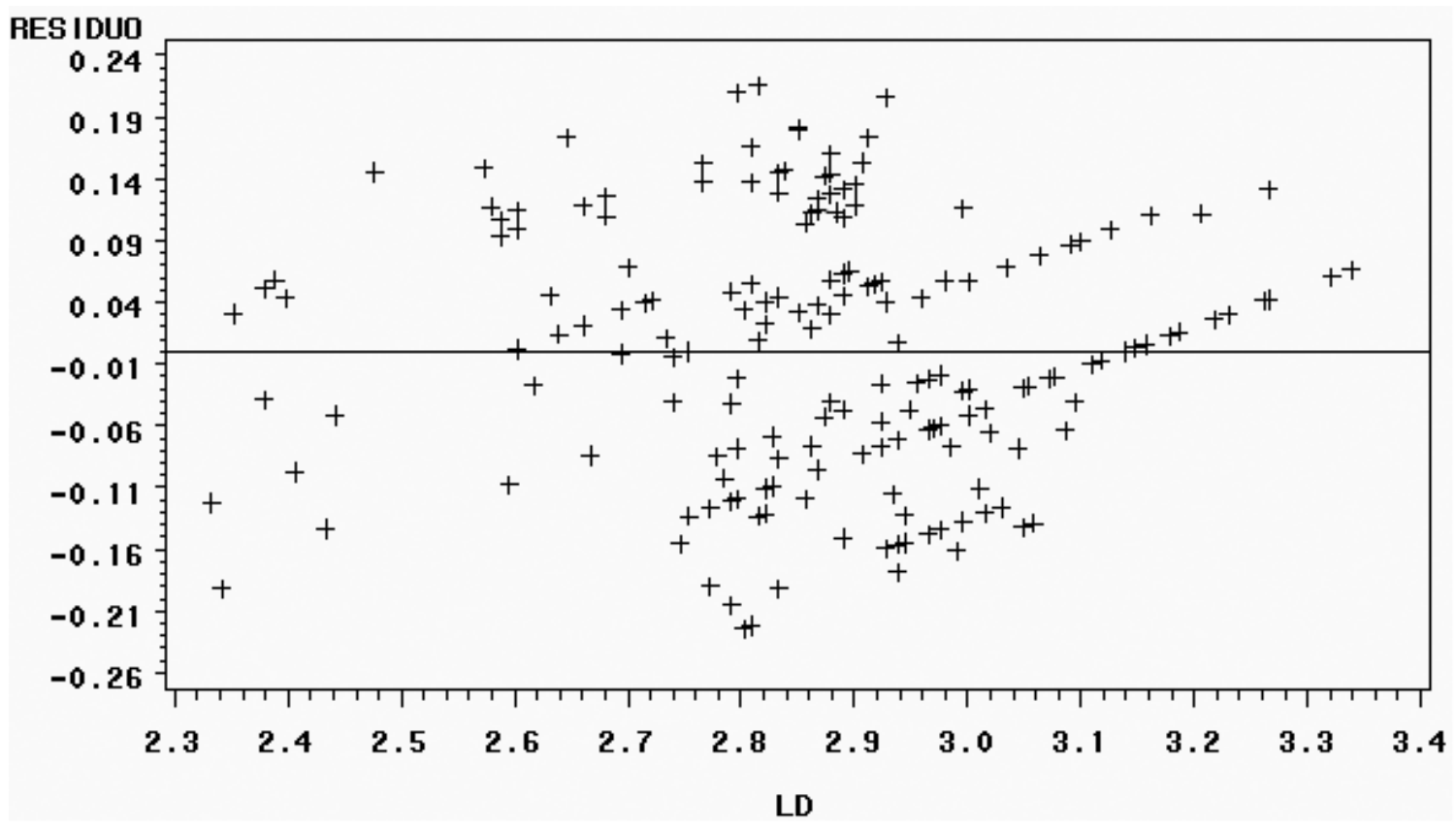

FIGURA 1: Distribuição dos resíduos da densidade de árvores por hectare pelo logaritmo do diâmetro (LD) para o modelo de Tang.

FIGURE 1: Residual distribution of tree density by hectare for logarithm of diameter (LD) for the Tang model.

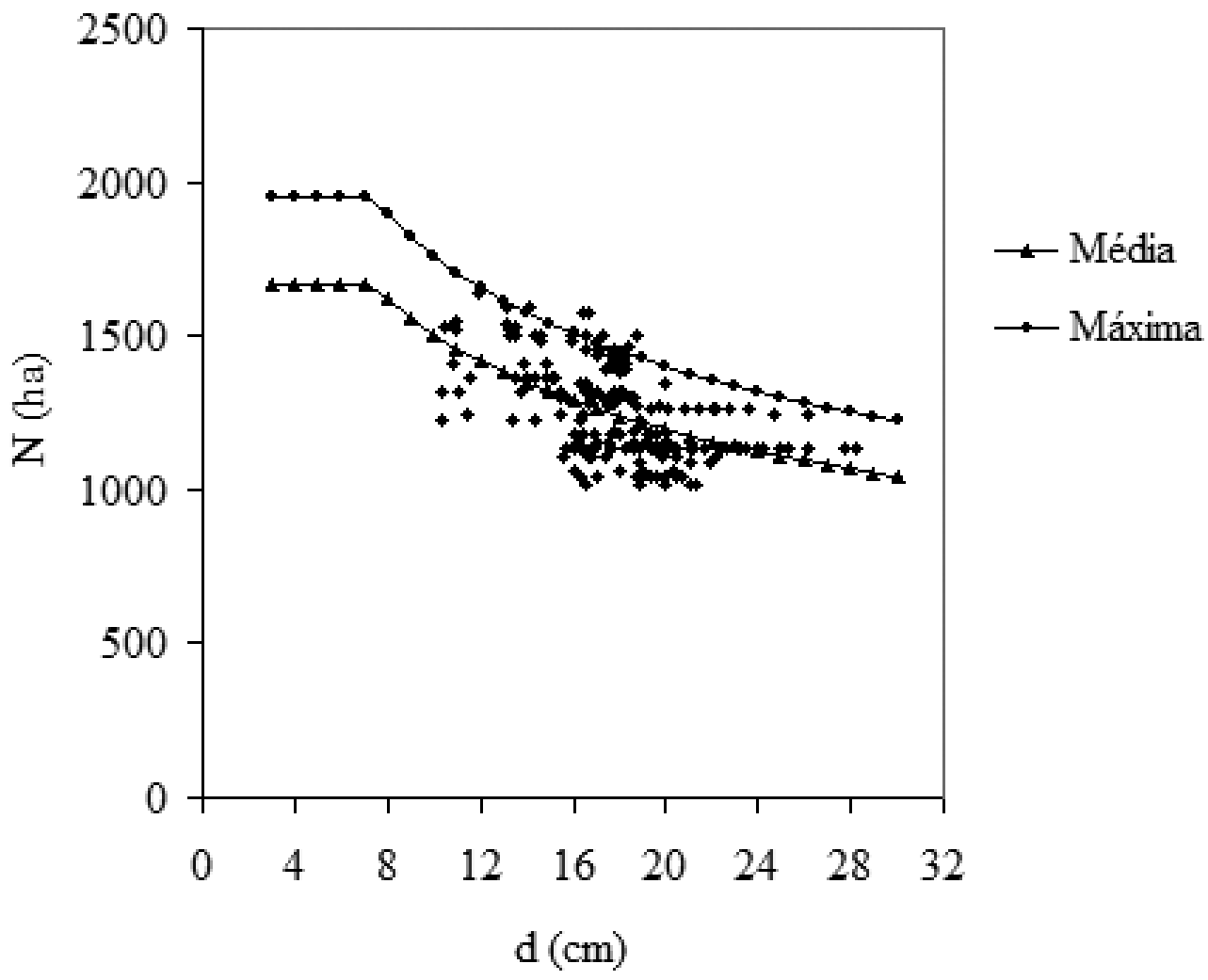

FIGURA 2: Número máximo e médio de árvores por hectare em função do diâmetro médio em povoamentos de Eucalyptus saligna, clone 4013, estimadas pelo modelo de Tang.

FIGURE 2: Maxinum and midlle number of trees in function of the diameter for Eucalyptus saligna, clone 4013, estimated by Tang model. 
e nutrientes do solo. À medida que estes elementos passam a ser mais escassos, devido à concorrência mais acentuada, os indivíduos mais debilitados ou dominados, naturalmente, vão sucumbindo da população. A partir deste momento começa a surgir com maior intensidade a mortalidade de indivíduos, se não houver intervenção com desbaste.

A densidade relativa da área basal por hectare em função do diâmetro, calculado pelo modelo proposto por Curtis (1982), apresentou um bom ajuste e precisão, com coeficiente de determinação ajustado igual a 0,73 e erro padrão da estimativa de 0,1589 , tendo resultado na expressão:

$$
G=0,3446997 \cdot\left(d^{1,5818}\right)
$$

Em que, $\mathrm{G}$ é a área basal por hectare e d o diâmetro médio da população.

No modelo o coeficiente angular 1,5818, determina o gradiente da linha que indica a velocidade do acréscimo da área basal por hectare com o aumento do diâmetro da população. Este parâmetro permitiu determinar a densidade relativa (DR) da população, através da expressão:

$$
D R=G /\left(d^{1,5818}\right)
$$

Logo, para uma determinada área basal por hectare e o diâmetro médio respectivo, numa idade, obtém-se o valor da densidade relativa (DR), que no caso resultou num valor de 0,3446 $(34,46 \%)$. Este valor corresponde à proporção da área basal em relação ao diâmetro médio num determinado momento de desenvolvimento. Então, considerando um diâmetro médio de $20 \mathrm{~cm}$, para a densidade relativa de $34,46 \%$, a área basal é igual a $39,4 \mathrm{~m}^{2} / \mathrm{ha}$. Desta forma, consegue-se projetar o desenvolvimento em área basal por hectare para diâmetros médios correspondentes, como pode ser observado na Figura 3.

$\mathrm{O}$ quociente entre o diâmetro pela área basal, expresso em função do diâmetro da população, mostra uma forma decrescente com o crescimento do diâmetro, devido ao aumento da densidade de área basal por hectare em relação ao diâmetro médio, como é demonstrado na Figura 4. Nesta relação é possível verificar que o crescimento em área basal por hectare é superior ao aumento do diâmetro, permitindo inferir que os povoamentos monoclonais de Eucalyptus saligna, clone 4013, podem ser manejados em alta densidade populacional, portanto, com desbastes de intensidade leve, isto possibilita a redução de perdas de produção volumétrica, com um significativo acréscimo em incremento diamétrico.

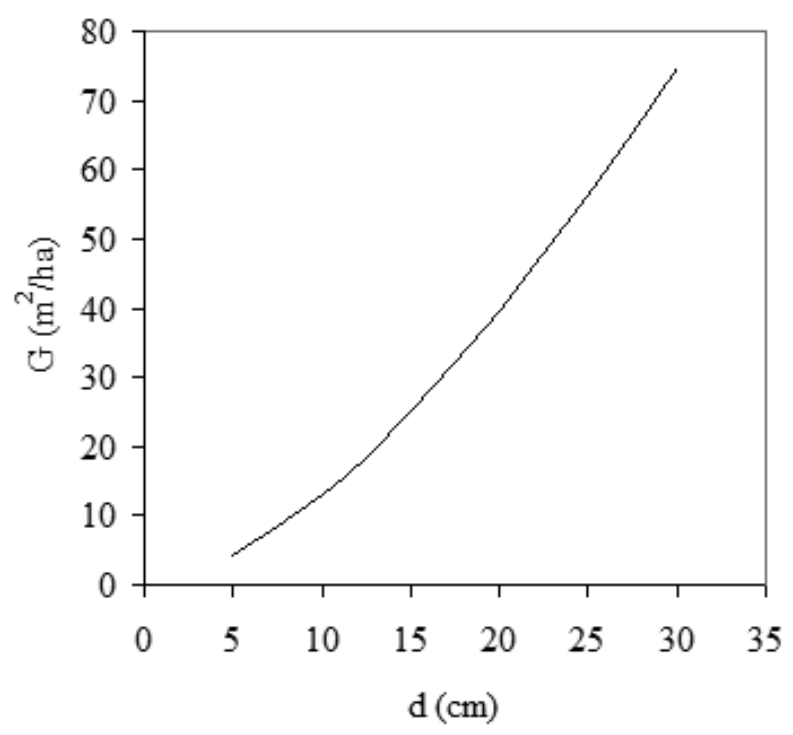

FIGURA 3: Desenvolvimento da área basal por hectare em função do diâmetro, com densidade relativa de $34,46 \%$ e $\mathrm{b}=1,5818$.

FIGURE 3: Development of the basal area for hectare in function of the diameter, with relative density of $34.46 \% \mathrm{e}$ $\mathrm{b}=1.5818$.

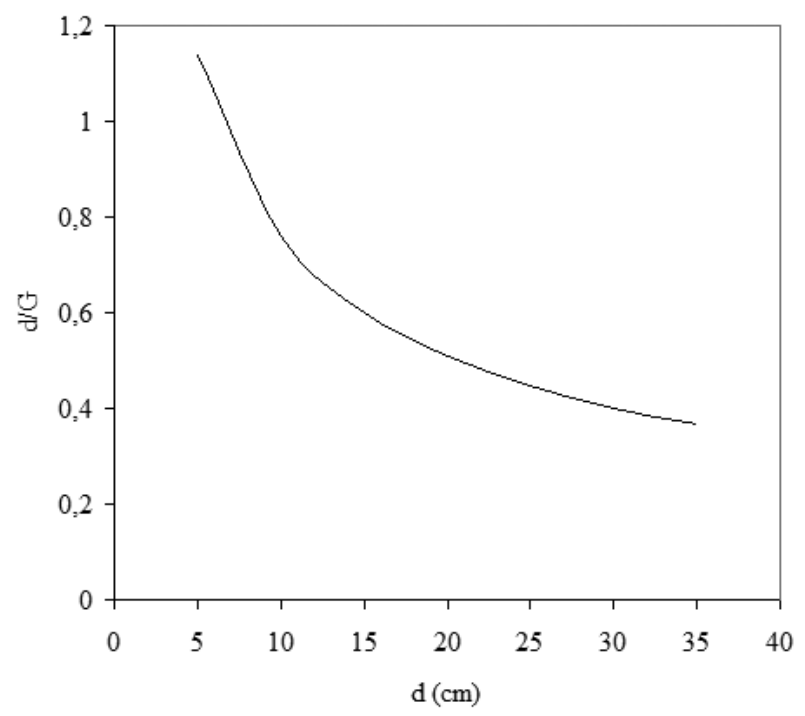

FIGURA 4: Quociente do diâmetro pela área basal por hectare como uma função do diâmetro.

FIGURE 4: The diameter and basal area for hectare with a diameter function. 
Num povoamento quando, ao manejar a população, for mantida uma área basal constante ocorre uma tendência de obter no tempo um povoamento subestocado. Isto, também pode ser verificado quando se mantém constante a proporção entre o diâmetro médio pela área basal por hectare $(\overline{\mathrm{d}}: \mathrm{G})$. Por conseguinte, em plantações manejadas de Eucalyptus saligna, quando for mantida constante a relação do diâmetro médio pela área basal, produzem-se árvores com crescimento livre. Porém, logo em seguida, com o crescimento em diâmetro e desenvolvimento da copa, surge um incremento de competição entre os indivíduos, o que se traduz em influência no crescimento diamétrico e na produção da população. Então, ao se manejar povoamentos de Eucalyptus saligna deve-se propiciar à população uma proporção $\overline{\mathrm{d}}: \mathrm{G}$ decrescente no tempo, à medida que forem sendo realizado os desbastes. Numa referência, quando um povoamento atingir um diâmetro médio de 10 $\mathrm{cm}$ a proporção $\overline{\mathrm{d}}: \mathrm{G}$ será aproximadamente 0,7 , diminuindo exponencialmente para um valor de 0,4 quando o diâmetro médio atingir $35 \mathrm{~cm}$.

Com o incremento corrente anual em diâmetro, tomado no período entre 14 a 15 anos, calculado com dados de parcelas permanentes em densidade completa, consegue-se estabelecer as zonas de competição, como é mostrado na Figura 5.

Desta forma, foram determinadas cinco zonas de concorrência distintas, definidas por: Zona de espaço excessivo, em que as árvores crescem livres da concorrência, onde o crescimento em altura está retardado; Zona de crescimento livre ocorre onde o incremento em diâmetro individual é máximo; Zona de aumento de competição ocorre quando o incremento individual em diâmetro das árvores começa a diminuir; Zona de estoque completo ocorre quando a produtividade do povoamento é máxima; Zona de iminente mortalidade surge quando começa haver mortalidade de indivíduos devido à densidade excessiva para um determinado diâmetro médio, o que é indesejável no manejo, pois o DR está próximo a 10. Entre as zonas de crescimento livre e a de estoque completo, situase, a zona de aumento de competição, onde o crescimento das árvores individuais começa a ser restringido pela competição. Estas linhas delimitam teoricamente as zonas de competição definidas em $50 \%, 25 \%$ e $12,5 \%$ do máximo, assumindo um DR $10(100 \%)$ na linha de máxima densidade populacional. Isto, igualmente foi determinado por outros pesquisadores, como Curtis (1982),

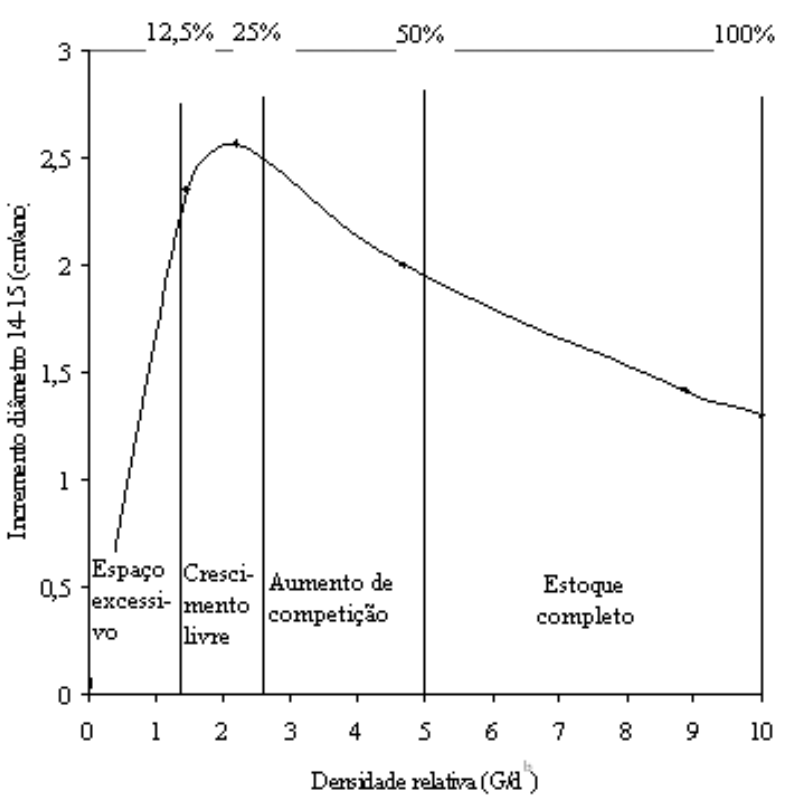

FIGURA 5: Incremento corrente anual em diâmetro entre 14 e 15 anos, para Eucalyptus saligna, clone 4013, em diferentes intensidades de manejo, indicando as zonas de competição $(b=1,5819)$.

FIGURE 5: Annual current increment in diameter by 14-15 years, for Eucalyptus saligna, clone 4013, in different management intensity, indicating competition zones $(\mathrm{b}=1.5818)$.

Archibald e Bowling (1995), Reid (2004) e outros.

O máximo incremento corrente anual em diâmetro ocorreu na zona de crescimento livre, entre as densidades relativas de 12,5 e $25 \%$, coincidindo com o que foi definido por Reid (2004). Com o aumento do índice de densidade relativa, acima de $25 \%$, inicia a competição entre as árvores sendo essa a zona que indica uma situação ótima de manejo, com uma densidade relativa em torno de $50 \%$. Acima desta zona ocorre uma severa competição entre as árvores, porém o estoque passa a ser máximo, quando se estabelece uma condição de mortalidade de indivíduos na população. Essa situação é indesejada no manejo, pois indica uma necessidade eminente de realização de desbaste.

\section{CONCLUSÕES}

Os resultados obtidos no presente trabalho permitiram concluir que:

a) O modelo de Tang apresentou uma boa eficiência e precisão na estimativa das densidades populacionais em função do diâmetro médio 
indicam que o autodesbaste inicia cedo, quando o diâmetro médio da população for $8 \mathrm{~cm}$, a densidade relativa foi igual a $34,46 \%$, com uma linha de gradiente igual a 1,5819 (b);

b) O máximo incremento corrente anual em diâmetro ocorreu entre as idades de 14 e 15 anos, entre as densidades relativas de 12,5 e $25 \%$, o que permitiu caracterizar a zona de crescimento livre de competição;

c) A zona de aumento da competição ocorreu entre as densidades relativas de 25 e $50 \%$; e zona de iminente mortalidade entre as densidades relativas de 50 e $100 \%$;

d) $\mathrm{O}$ modelo de autodesbaste permitiu detectar cinco zonas de concorrência ao longo do desenvolvimento dos povoamentos: espaço excessivo, crescimento livre, aumento de competição, estoque completo e iminente mortalidade, em relação à densidade relativa da população.

\section{AGRADECIMENTOS}

Os autores agradecem ao Eng. Jorge Mayer Klein e a empresa Celulose Riograndense, pela cessão de áreas para instalação do experimento e os dados dendrométricos para realização do presente estudo.

\section{BIBLIOGRAFIAS CONSULTADAS}

ARCHIBALD, D. J.; BOWLING, C. Jack pine density management diagram for boreal Ontario. Northeast Science \& Technology, NEST Technical Note TN-005, 1995, 19 p.

BOROUGH, C. J. et al. Yield statistics. In: HILLIS, W. E., BROWN, A. G. (eds), Eucalyptus for wood production. CSIRO/Academic Press, p.1-225, 1984.

BREDENKAMP, B. V.; BURKHART, H. E. An examination of spacing indices for Eucalyptus grandis. Canadian Journal of Forest Research. New Westminster, v. 20, p. 1909-1916, July, 1990.

CELLINI, J. M. et al. Modelos de perfil de tronco en Nothofagus pumilio (Polp, et Endl,) Krasser y su utilización en el cálculo del volumen total. Invest. Agr,: Sist. Recur. For. Madrid, n. 2, v. 11, p. 245261, 2002.

CURTIS, R. O. A simple index of stand density for Douglas-fir. Forest Science, Bethesda, n. 1, v. 28, p. 92-94, Mar. 1982.

DEBELL, D. S.; WHITESELL, C. D. Diameter- density relationships provide tentative spacing guidelines for Eucalyptus saligna in Hawaii. United States Department of Agriculture. Berkeley, 1988, 3 p.

DEL RIO, M.; MONTERO, G.; BRAVO, F. Analysis of diameter-density relationships and self-thinning in non-thinned even-aged Scots pine stands. Forest Ecology and Management. Amsterdan, v. 142, p. 79-87, Mar. 2001.

EMBRAPA. Sistema brasileiro de classificação de solos. Brasília: Embrapa, 1999, 412 p.

LAASASENAHO, J.; KOIVUNIEMI, J. Dependence of some stand characteristics on stand density. Tree Physiology. Oxford, v. 7, p. 183-187, 1990.

MORENO, J. A. Clima do Rio Grande do Sul. Porto Alegre: Secretaria da Agricultura - Diretoria de Terras e Colonização, 1961, 42 p.

OSAWA, A.; ALLEN, R. B. Allometric theory explains self-thinning relationships of mountain beech and Red pine. Ecology. n. 74, v. 4, p. 10201032, Apr. 1993.

PALAHI, M.; MIIMA, J.; MONTERO, E. Standlevel yield model for scots pine (Pinus sylvestris L, ) in north-east Spain. Invest. Agr. Sist. Recr. For. Madrid, v. 11, n. 2, p. 409-424, 2002.

REID, R. Diameter-basal area ratio as a practical stand density measure for pruned plantations. Forest Ecology and Management. Amsterdan, v. 233, p. 375-382, Sept. 2006.

REID, R. Silvicultural management of Australian blackwood (Acacia melanoxylon) in plantations and multi-purpose forests. In: GOVE, J. H. Structural stocking guides: a new look at an old friend. National Research Council Canada. Ottawa, v. 34, n. 5, May, 2004.

REINEKE, L. H. Perfecting a stand-density index for even-aged forests. Canadian Agricultural Research. Ottawa, n. 46, p. 627-638, 1933.

SAS Institute Inc. SAS/STAT user's guide. Version 8 (computer manual), SAS Institute Inc., Cary, N.C. 1999.

SMITH, D. J.; WOODS, M. E. Red pine and white pine density management diagrams for Ontario. Ontario: Ministry of Natural Resources, Southcentral Sciences Division, Sault Ste, Marie, ON, 1997, 31 p. (Tech, Rep, n.48)

SMITH, N. J.; HANN, D. W. A growth model based on the self-thinning rule. Canadian Journal of Forest Research. New Westminster, v. 16, n. 2, p. 330-334, Apr. 1986.

STERBA, H.; MONSERUD, R. A. The maximum 
density concept applied to uneven-aged mixedspecies stands. Forest Science. Bethesda, v. 39, n. 3, p. 432-452, Aug. 1993.

TANG, S. et al. A growth and self-thinning model for pure even-aged stands: theory and applications. Forest Ecology and Management. Amsterdan, v. 70, p. 67-73, Dec. 1994.

TANG, S.; MENG, F. R.; MENG, C. H. The impact of initial stand density and site index on maximum stand density index and self-thinning index in a stand self-thinning model. Forest Ecology and Management. Amsterdan, v. 75, p. 61-68, June, 1995.

WEST, P. W.; BOUROUGH, C. J. Tree supervision and the self-thinning, Rule in a monoculture of Pinus radiata D,Don. Annals of Botany. Oxford, v. 52, p. 149-158, 1983.

WESTOBY, M. The place of the self-thinning rule in population dynamics. American Naturalist. v. 118, n. 4, p. 581-587, Oct. 1981.

YODA, K. et al. Self-thinning in over crowed pure stands under cultivated and natural conditions. Jor. Biol. Osaka City Univ. Osaka, v. 14, p. 107-129, 1963.

ZEIDE, B. A relationship between size of trees and their number. Forest Ecology and Management. Amsterdan, v. 72, p. 265-272, Apr. 1995.

ZEIDE, B. Analysis of the $3 / 2$ power law of selfthinning. Forest Science. Bethesda, v. 33, n. 2, p. 17-537, June, 1987.

ZEIDE, B. Self-thinning and stand density. Forest Science. Bethesda, v. 37, n. 2, p. 517-523, June, 1991.

ZEIDE, B. Tolerance and self-tolerance of trees. Forest Ecology and Management. Amsterdan, v. 13, p. 149-166, Nov. 1985. 